Documentation et bibliothèques

DOCUMENTATION BIBLIOTHEQUES

\title{
Publications officielles québécoises - Le journal des Débats (II)
}

\section{Gaston Bernier}

Volume 21, numéro 4, décembre 1975

URI : https://id.erudit.org/iderudit/1055382ar

DOI : https://doi.org/10.7202/1055382ar

Aller au sommaire du numéro

\section{Éditeur(s)}

Association pour l'avancement des sciences et des techniques de la documentation (ASTED)

\section{ISSN}

0315-2340 (imprimé)

2291-8949 (numérique)

Découvrir la revue

\section{Citer cet article}

Bernier, G. (1975). Publications officielles québécoises - Le journal des Débats (II). Documentation et bibliothèques, 21(4), 197-200.

https://doi.org/10.7202/1055382ar

\section{Résumé de l'article}

La publication du journal des Débats de l'Assemblée nationale du Québec remonte à 1964 et est le fruit d'une longue recherche. L'auteur fait l'historique de ce compte rendu officiel ainsi que des différentes expériences tentées à l'époque de la Confédération et à la fin du XIXe siècle. Il souligne également les recherches menées actuellement pour combler les périodes où il n’y a pas eu de compte rendu officiel des délibérations.
Tous droits réservés (C) Association pour l'avancement des sciences et des techniques de la documentation (ASTED), 1975
Ce document est protégé par la loi sur le droit d'auteur. L’utilisation des services d'Érudit (y compris la reproduction) est assujettie à sa politique d'utilisation que vous pouvez consulter en ligne.

https://apropos.erudit.org/fr/usagers/politique-dutilisation/ 


\title{
Publications officielles québécoises - Le journal des Débats (II)
}

\author{
Gaston Bernier* \\ Bibliothèque nationale \\ Abidjan, Côte d'Ivoire
}

La publication du journal des Débats de l'Assemblée nationale du Québec remonte à 1964 et est le fruit d'une longue recherche. L'auteur fait l'historique de ce compte rendu officiel ainsi que des différentes expériences tentées à l'époque de la Confédération et à la fin du XIXe siècle. II souligne également les recherches menées actuellement pour combler les périodes où il n'y a pas eu de compte rendu officiel des délibérations.

The publication of the journal des Débats exists since 1964 and results from a long investigation. The author makes the historical account of the official report in the same manner as the different experiences atempted in the times of Confederation and at the end of the 19th Century. He mentions as well the actual searches accomplished to refill the periods when there was no official report of the deliberations.

La publicación del journal des Débats de la Asemblea Nacional de Québec remonta al año 1964 y resulta de una larga investigación. El autor hace la reseña histórica de este informe oficial de mismo modo que de las diferentes experiencias atentadas en la época de la Confederación y a finales del siglo 19. Menciona también las búsquedas efectuadas actualmente para rellenar los períodos durante los cuales no hubo informe oficial de las deliberaciones.

Les bibliothécaires se rappelleront avoir dû répondre à un usager que le journal des Débats n'existe que depuis 1964 . Ordinairement, l'usager a peine à croire que ce compte rendu n'existe que depuis cette date et qu'il soit un legs de ce qu'on a appelé la Révolution tranquille!

La réalisation du journal des Débats a été précécrée de trois années d'étude et d'essai. Le gouvernement de Jean Lesage, élu en 1960, mit sur pied, au début de 1961, un "comité formé pour étudier le projet d'établir un journal des Débats». Présidé par le Président de l'Assemblée législative d'alors, Lucien Cliche, le comité fit rapport le 9 juin. 1 On recommandait l'adoption d'une loi pour «instituer un journal des Débats reproduisant les discours dans la langue dans laquelle ils

* N.D.L.R.: Jusqu'à tout récemment, Gaston Bernier travaillait au service de référence de la Bibliothèque de la Législature de l'Assemblée nationale du Québec.

1. Journaux de l'Assemblée législative, vol. 96 (1960/61), 970-971. ont été prononcés» et pour autoriser le Président de l'Assemblée législative ou le gouvernement «à passer les contrats nécessaires pour assurer l'enregistrement des débats et leur publication". ${ }^{2}$

Le comité présenta, au cours de la session de 1962 , un deuxième rapport. ${ }^{3}$ On y soulignait le fait que "tous les membres sont d'accord sur la nécessité, dès cette session, d'établir un système de reportage des débats par voie de sténographie". ${ }^{4}$ On notait également que les retards étaient causés par les difficultés à trouver des locaux convenables.

En dépit des obstacles, l'enregistrement des débats de la première session de la vingtseptième législature fut réalisé au complet de même que celui de la courte session de l'été 1963. La publication de ces comptes rendus

2. Ibid., 971.

3. Journaux de l'Assemblée législative, vol. 97 (1962), 179-180.

4. Ibid., 180. 
verbatim fut distribuée à très peu d'exemplaires. Quelques bibliothèques du Québec possèdent la collection expérimentale ${ }^{5}$ : la $\mathrm{Bi}$ bliothèque nationale, la bibliothèque de I'Université Laval et celle de l'Assemblée nationale. On publia également les débats de la deuxième sesśion. ${ }^{6}$ Dans ce dernier cas, le volume se présente sous la forme actuelle du journal des Débats, mais il ne possède ni mention du volume, ni numérotation.

Officiellement, le journal des Débats est né avec la session de 1964 et la première séance dont le compte rendu existe est celle du 14 janvier. La publication a paru régulièrement depuis?.

5. Débats, 1ère session, 27 e législature (15 janvier 1963-1 1 juillet 1963), 56 vol. (Polycopié).

6. Débats de l'Assemblée législative du Québec, $2 \mathrm{e}$ session, 27e législature (21, 22 et 23 août 1963), Québec, Imprimeur de la reine, 1963. 205 p.

7. Vol. 1 (3e session, 27e législature; 14 janvier 1964 31 juillet 1964), 5,240 p.

Vol. 2 (4e session, 27 e législature; 21 janvier 19656 août 1965), 5,012 p.

Vol. 3 (5e session, 27 e législature; 22 octobre 1965), $98 \mathrm{p}$.

Vol. 4 (6e session), 27 e législature; 25 janvier 1966-31 mars 1966), 1,920 p.

Vol. 5 (1ère session, 28 e législature; 1er décembre 1966-12 août 1967), 5,329 p.

Vol. 6 (2e session, 28e législature; 20 octobre 1967), $83 \mathrm{p}$.

Vol. 7 (3e session, 28e législature; 20 février 19685 juillet 1968 et 22 octobre-18 décembre 1968), $5,174 \mathrm{p}$.

Vol. 8 (4e session, 28 e législature; 25 février 1969-13 juin 1969 et 7 octobre-23 décembre 1969), 5,580 p. Vol. 9 (5e session, 28 e législature; 24 février 1970-11 mars 1970), $325 \mathrm{p}$.

Vol. 10 (1ère session, 29e législature; 9 juin 1970-19 décembre 1970), 2,634 $\mathrm{p}$.

Vol. 11 (2e session, 29e législature; 23 février 1971-24 décembre 1971), 5,775 p.

Vol. 12 (3e session, 29e législature; 7 mars 1972-14 mars 1973), 4,395 $\mathrm{p}$.

Vol. 13 (4e session, 29e législature; 15 mars 1973-6 juillet 1973), 2,326 $\mathrm{p}$.

Vol. 14 (1ère session, 30e législature; 22 novembre 1973-22 décembre 1973), $981 \mathrm{p}$.

Vol. 15 (2e session, 30e législature; 14 mars 1974-).
La publication de la relation textuelle des débats depuis 1964 n'était pas cependant la première tentative de doter l'Assemblée nationale d'un tel compte rendu.

Au cours de la seconde moitié du XIXe siècle ${ }^{8}$, le journaliste Roch-Pamphile Vallée publia sept livraisons de quatre pages d'un hebdomadaire auquel il donna le nom d'Echo de la session, journal des débats parlementai$r^{r e s}{ }^{9}$. La période ainsi ouverte va du 7 novembre au 23 décembre 1871. L'Écho reproduit, dans certains cas, les discours intégraux des députés, mais se contente parfois d'un bref résumé.

Après l'expérience sans lendemain de $\mathrm{Val}$ lée, c'est Gabriel-Alphonse Desjardins, N. Malenfant et Louis-Georges Desjardins qui publièrent successivement un compte rendu des débats pour la période qui va de 1879 à 1893 inclusivement ${ }^{10}$.

8. André Beaulieu, "Les Débats de l'Assemblée nationale». Bibliothèque de la Législature du Québec. Bulletin trimestriel, vol. 2, no 4 (octobre 1971), 23-30.

9. L'Écho de la session; journal des Débats parlementaires, no 1 (13 novembre 1871) - no 7 (26 décembre 1871), $28 \mathrm{p}$.

10. Débats de la Législature provinciale de la province de Québec, 4e législature, 2e session (1879) - 8e législature, 2e session (1893), Québec, 1879-1895. 15 vol. (Le titre devint Débats de la Législature de la province de Québec en 1882 et changea de nouveau pour les deux derniers volumes: Débats de l'Assemblée législative de la province de Québec). 
Ces compilations n'incluent pas uniquement les débats de la Chambre basse ou Assemblée législative. Elles comprennent également le compte rendu du Conseil législatif ou Chambre haute jusqu'en 1886 inclusivement. Á compter de 1887 et jusqu'en 1895, C.-E. Rouleau compila et publia séparément les débats des conseillers législatifs".

Ces compilations, comme celles publiées pour la Chambre des Communes du Canada jusqu'en 1880 ou celles de la Chambre des Communes de Grande-Bretagne, avant 1909, ne sont pas des comptes rendus officiels. Cependant, dans le cas qui nous préoccupe, il faut souligner que les pages de titres des volumes 7 à 11 (1885 à 1889) portent la mention: "Publiés sous les auspices de la Législature de la province de Québec». D'ailleurs, Desjardins reçut des subventions gouvernementales de l'ordre de $\$ 4,000$ à $\$ 6,000$ pour leur publication et c'est même le refus du premier ministre Mercier qui causa l'arrêt des Débats Desjardins'2.

Par la suite, N. Malenfant et Louis-Georges Desjardins, ce dernier frère d'Alphonse, continuèrent la collection jusqu'en 1893. Malenfant publia les comptes rendus des deux sessions de 1890 (vol. 12 et 13 ) et L.-G. Desjardins ceux des sessions de 1892 et de 1893. La tâche devenait sans doute trop lourde et trop coûteuse. Le volume couvrant la session de 1893 parut en 1895 seulement. Et les textes des débats des deux sessions suivantes, déjà réunis, ne furent pas publiés.

Cette collection présente de nombreuses lacunes, comme d'ailleurs la tentative précédente de Vallée. Les Débats Desjardins reprennent, selon Marcel Hamelin, «à peu près intégralement la version des débats parlementaires parue dans Le Canadien". Cette origine unique et l'absence de contrôle à l'aide d'autres sources ne permet pas de conclure à l'impartialité ou, tout simplement, à l'authenticité du texte. De plus, ils ne constituent pas un compte rendu intégral de tous les discours, Desjardins établissant un résumé des discours prononcés à partir de notes prises en sténographie $^{13}$.

L'échec des tentatives de 1871 et de 18791895 ne doit pas surprendre outre mesure.

11. Débats du Conseil législatif de la province de Québec, 6e législature, 1ère session (1887) - $8 e$ législature, 5e session (1895), Québec, 1887-1895. 10 vol.

12. Yves Roby, Alphonse Desjardins et les Caisses populaires, 1854-1920, Montréal, Fides, 1964, 14-17.

13. Ibid., 15
Dans l'ensemble, il n'était pas coutume, avant 1867, de publier les comptes rendus verbatim des délibérations des assemblées parlementaires en Amérique du Nord, même si certaines colonies (lle-du-Prince-Édouard, NouvelleÉcosse) en possédaient. Dans la province du Canada, la première publication du genre fut le compte rendu officiel du débat sur la Confédération ${ }^{14}$ qui eut lieu au cours de la session de 1865. Le Hansard fédéral canadien ne vit vraiment le jour que le 4 mai 1880 alors que la Chambre engagea son propre personnel ${ }^{15}$. Toutefois, le Hansard était publié sous contrat par l'entreprise privée ${ }^{16}$ depuis 1875.

Un second facteur a également joué contre la création d'un "hansard" québécois: l'excellence des reportages de la presse quotidienne. P. B. Waite, l'auteur de la reconstitution des Débats de la Chambre des Communes, $1867 / 68$, souligne ce fait ${ }^{17}$ tout en le regrettant, car l'intérêt porté aux délibérations de l'Assemblée législative par la presse de l'époque fit que les reportages des activités du Parlement fédéral furent relégués au second plan. Un second historien, Marcel Hamelin, porte un témoignage identique dans l'introduction à la compilation des délibérations du Parlement québécois que l'Éditeur du

14. Débats parlementaires sur la question de la Confédération des provinces de l'Amérique britannique du nord. 3e session, 8e parlement provincial du Canada, Québec, Hunter, Rose et Lemieux, 1865, IX, 1027 p. ( $L$ 'index suivant facilitera la consultation de ce volume: Archives publiques du Canada. Division des publications. Index aux débats parlementaires sur la Confédération...; compilateur: M.A. Lapin, Éditeur: J.S. Patrick. Ottawa, Imprimeur du roi, 1952. 71 p.)

15. Le lecteur intéressé à l'évolution des idées et des faits concernant les comptes rendus verbatim pourra consulter le savant article suivant: Elisabeth Nish, "Canadian Hansard 1841: interpreting the canadian parliamentary press ", in Debates of the Legislative Assembly of United Canada; 1841. Montréal, Presses de l'École des hautes études commerciales, 1970, IX-Cl.

16. Ibid., XLVIII.

17. Débats de la Chambre des Communes; $1867 / 68$, 1 ère session, 1ère législature, Ottawa, Queen's printer, 1.968, VIII. 
journal des Débats a publiée à la fin de 1974. C'est dire que la population du Québec, de même que les hommes politiques, pouvait suivre, sans grand problème, les activités de ses parlementaires.

Si le besoin d'un compte rendu verbatim ne semble pas avoir été ressenti très fortement avant 1900 et même avant la "Révolution tranquille ${ }^{18}$, depuis quelques années une vague de reconstitutions se répand. Le centenaire du British North American Act (BNA) - Acte de l'Amérique du nord britannique en 1967 inspira à Erik Spicer, bibliothécaire du Parlement du Canada, l'idée d'une reconstitution des débats ayant eu lieu à la Chambre des Communes entre 1867 et 1874 . Le premier volume ${ }^{19}$ parut en 1968 . Les premières délibérations du Sénat fédéral furent également compilées et publiées ${ }^{20}$ mais les volumes suivants ne sont toujours pas publiés au début de 1975. II semble cependant que les comptes rendus des délibérations de l'année 1869 paraîtront avant la fin de l'année.

Le second projet actuelleḿnent en cours, dirigé par Elisabeth Nish, porte sur la période de 1841 à 1867. Déjà deux volumes sont apparus sur le marché ${ }^{21}$.

Inspirés sans doute par ces deux entreprises, Marcel Hamelin et André Beaulieu ont à leur tour mis sur pied une équipe de recherche

18. Si l'on excepte les comptes rendus de divers "Comités" parlementaires, la seule publication ayant vu le jour entre 1895 et 1963 est la suivante: Quebec and Confederation; a record of the debate of the Legislative assembly of Quebec on the motion proposed by J.-N. Francœur, member for Lotbinière, Quebec and Ottawa Press Galleries, 1918, 136 p.

19. Débats de la Chambre des Communes, 1ère session, 1ère législature, $1867 / 1868$, Ottawa, Queen's printer, 1968, XX, $854 \mathrm{p}$

20. Debates of the Senate, 1st session, 1st parliament, 1867/68, Ottawa, 1968, $392 \mathrm{p}$

21. Debates of the Legislative Assembly of United Canada, 1841-1867, vol. 1 - Montréal, Presses de l'École des hautes études commerciales, 1970 - qui travaille à la reconstitution des débats de l'Assemblée législative du Québec. La période à combler s'étend de 1867 à 1963. C'est dire l'ampleur du travail. Les auteurs ne reprendront pas cependant les comptes rendus réalisés par Desjardins-Malenfant-Desjardins (de la 2 e session de la 4 e législature à la 2 e session de la $8 e$ législature inclusivement). Déjà on a publié le premier volume de la collection ${ }^{22}$ : il couvre la première législature (1867/68 à 1870).

L'engouement actuel pour la reconstitution des débats trouve son explication dans l'utilité de telles publications. Ce facteur explique également l'existence à peu près systématique et automatique des comptes rendus verbatim courants dans les provinces du Canada ${ }^{23}$.

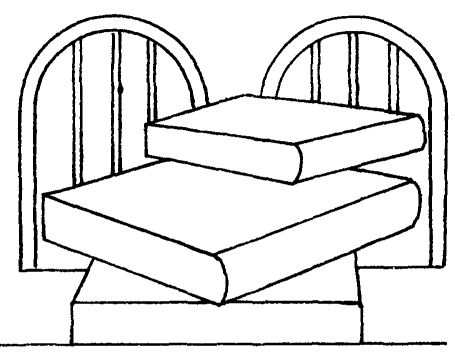

22. Débats de l'Assemblée législative, lère législature, 1ère session, 1867/68 - Texte établi par Marcel Hamelin, Québec, Assemblée nationale. Journal des Débats, 1974 -

23. Voir les inventaires dont le signalement a été donné au début du premier article de la série: Documentation et bibliothèques, vol. XX, no 4 (décembre 1974), 173 Également A. Paul Pross et Catherine A. Pross, Government Publishing in the Canadian Provinces; a Prescriptive Study, Toronto, University of Toronto Press, 1972, p. 116-117. 